\title{
Simulated operation combines patient-specific guide technology is superior to conventional technology for supramalleolar osteotomy: a retrospective comparative study
}

\section{Chenggong Wang}

Xiangya Hospital Central South University

\section{Can Xu}

Xiangya Hospital Central South University

Yusheng Li

Xiangya Hospital Central South University

Mingqing Li

Xiangya Hospital Central South University

\section{Da Zhong}

Xiangya Hospital Central South University

\section{Long Wang}

Xiangya Hospital Central South University

Hua Liu ( $\square$ dr_liuhua_xyyy@126.com )

Xiangya Hospital Central South University https://orcid.org/0000-0003-2034-6407

\section{Research article}

Keywords: Supramalleolar osteotomy, Simulated operation, Patient-specific guide, Accuracy of preoperative planning, Practicability of application

Posted Date: March 27th, 2020

DOI: https://doi.org/10.21203/rs.3.rs-18783/v1

License: (9) This work is licensed under a Creative Commons Attribution 4.0 International License. Read Full License 


\section{Abstract}

Background: Over the past seven years, our team has designed a simulated operation combines patientspecific guide (SO-PSI) assisted supramalleolar osteotomy (SMOT) method and applied it to the clinic. This study aimed to determine the difference between SO-PSI technology and conventional operation (CO) technology for SMOT in preoperative planning, intraoperative application and postoperative curative effect.

Methods: We retrospectively analyzed the data of SMOT in our hospital between October 2014 and December 2018; 28 patients were enrolled and divided into CO group $(n=17)$ and SO-PSI group $(n=11)$; the mean follow-up time was 33.4 (range, 13 to 59) months. We analyzed and compared perioperative data, accuracy of preoperative planning and intraoperative application, difference between pre- and postoperative of radiologic ankle angles, and change of AOFAS score, VAS, range of motion of ankle and Takakura stage after operation. Then we performed statistical analyses.

Results: All ankle alignments and positions of two groups have been recovered. Compared with $\mathrm{CO}$ group, SO-PSI group has a shorter operative time and a postoperative hospital stay, a decreased number of fluoroscopies and a reduction of albumin, a longer preoperative planning time and a preoperative hospital stay and an increased hospitalization expenses. In SO-PSI group, the ankle angles between preoperative planning and postoperative shown good correlation, but not in $\mathrm{CO}$ group; the mean tibial ankle center discrepancy of SO-PSI group was $1.86 \pm 1.06 \mathrm{~mm}$. After follow-up, all radiologic parameters of two groups significantly improved, but the improvement of tibial anterior surface angle and tibiotalar tilt angle of SOPSI group were more obvious than that of CO group; AOFAS score, VAS, range of motion of ankle and Takakura stage of two groups improved after operation; but overall the improvements of SO-PSI group were better than the $\mathrm{CO}$ group.

Conclusions: SO-PSI technology can carry out the preoperative plan accurately and quickly in SMOT; In general, compared with the conventional technology, SO-PSI technology has advantages in preoperative planning, intraoperative application and postoperative curative effect. Keywords: Supramalleolar osteotomy; Simulated operation; Patient-specific guide; Accuracy of preoperative planning; Practicability of application

\section{Background}

Supramalleolar osteotomy (SMOT) is an effective procedure for the treatment of midstage asymmetric ankle osteoarthritis [1-4]. Related studies have proved that the key factors affecting the postoperative curative effect of SMOT are the postoperative ankle Takakura stage [5], lower limb power line [6] and ankle angles [7]; but SMOT uses conventional operation (CO) method often does not perfectly accomplish these surgical aims leading to some patients have unsatisfactory outcomes $[8,9]$. The reasons include preoperative planning dependence on freehand drawing, and surgeons determine the operative procedure all depend on the surgeon's experiences and fluoroscopies [10]. 
Some studies have demonstrated that simulated operation combines patient-specific guide (SO-PSI) can optimize preoperative planning and operative process, and make operation personalized and precision [11-13]. Over the past seven years, our team has designed a SO-PSI assisted SMOT method and applied it to the clinic. The surgeons can complete preoperative evaluation and operation simulation on threedimensional (3D) digital model, and get the best planning after repeated confirmation; then accurately execute the preoperative planning with the help of PSI during the operation.

Therefore, we collected the data of preoperative planning; intraoperative application and postoperative follow-up of SMOT performed by $\mathrm{CO}$ and SO-PSI technology, and conducted a retrospective comparative study to determine whether CO or SO-PSI was more effective.

\section{Methods}

\section{Study design and patients}

This study has been approved by the Ethics Committee of our hospital. We retrospectively reviewed the clinical and imaging data of patients with SMOT used or did not used SO-PSI between October 2014 and December 2018. The inclusion criteria were as follows: (1) more than 18 years old; (2) primary surgery; (3) unilateral SMOT; (4) with Takakura stage II, IIIA or IIIB; (5) with clinical symptoms, like pain, and limitation of daily activities; (6) treated with SMOT with at least one year follow-up. The exclusion criteria were (1) refused to participate the study; (2) Charcot arthropathy, rheumatoid arthritis or ankle infection; (3) physical activity disorders caused by other disease, such as neurologic disorders; (4) mental illness.

In the end, 28 patients were included; we divided them into CO group $(n=17)$ and SO-PSI group $(n=11)$. Basic information of the patients is presented in Table 1 ; the demographics and preoperative situation of two groups have no significant difference. Follow-up was conducted at 3,6 , and 12 months postoperatively, and at least one follow-up after postoperative 12 months. The mean follow-up time was 33.4 (range, 13 to 59 ) months. 
Table 1

Basic information of the patients.

\begin{tabular}{|c|c|c|c|}
\hline & $\mathrm{CO}$ group & SO-PSI group & P values\# \\
\hline Number of patients & 17 & 11 & \\
\hline Gender (male/female) & $5 / 12$ & $4 / 7$ & 0.700 \\
\hline Mean of Age (years) & $46.4 \pm 11.6$ & $53.8 \pm 14.4$ & 0.147 \\
\hline Mean of BMI $\left(\mathrm{kg} / \mathrm{m}^{2}\right)$ & $24.8 \pm 4.4$ & $25.6 \pm 4.6$ & 0.622 \\
\hline \multicolumn{4}{|l|}{ Etiology (No. and \%) } \\
\hline Osteoarthritis & $4(23.5 \%)$ & $3(27.3 \%)$ & 0.823 \\
\hline Posttrauma arthritis & $13(76.5 \%)$ & $8(72.7 \%)$ & 0.823 \\
\hline Diabetes (No. and \%) & $2(11.8 \%)$ & $2(18.2 \%)$ & 0.636 \\
\hline Smoker (No. and \%) & $3(17.6 \%)$ & $4(36.4 \%)$ & 0.264 \\
\hline \multicolumn{4}{|l|}{ Takakura stage (No. and \%) } \\
\hline Takakura II & $3(17.6 \%)$ & $1(9.1 \%)$ & 0.527 \\
\hline Takakura IIIA & $9(52.9 \%)$ & $5(45.5 \%)$ & 0.699 \\
\hline Takakura IIIB & $5(29.4 \%)$ & $5(45.5 \%)$ & 0.387 \\
\hline Mean of pre-op AOFAS score & $52.5 \pm 10.6$ & $51.7 \pm 10.9$ & 0.860 \\
\hline Mean of pre-op VAS & $7.7 \pm 1.4$ & $7.3 \pm 1.6$ & 0.463 \\
\hline Mean of pre-op ROM of ankle $\left({ }^{\circ}\right)$ & $31.2 \pm 6.8$ & $31.8 \pm 8.4$ & 0.850 \\
\hline Mean of follow-up time (months) & $28.6 \pm 12.4$ & $35.9 \pm 12.9$ & 0.150 \\
\hline \multicolumn{4}{|c|}{$\begin{array}{l}\text { Abbreviations: CO: Conventional operation group; SO-PSI: Simulated operation combines Patient- } \\
\text { specific guide; BMI: body mass index; AOFAS score: the American Orthopedic Foot and Ankle Societ } \\
\text { score; VAS: visual analogue scale; ROM: range of motion; pre-op: preoperative. }\end{array}$} \\
\hline
\end{tabular}

\section{Preoperative evaluation and planning}

In the $\mathrm{CO}$ group, preoperative planning was based on radiography images in combination with the surgeon's experience. First, we drew anatomical axis of tibia and angles of ankle (Figs. 1a and 1b), such as tibial anterior surface angle (TAS), tibiotalar tilt angle (TTA), malleolar angle (MA) and tibial lateral surface angle (TLS); then we drew osteotomy plan on the X-ray, the aim of osteotomy was to make the 
ankle angles ideal while keeping the force line correct [7, 14-17] (Fig. 1c). However, only target value of TAS and TLS can be accurately designed in preoperative plan.

In the SO-PSI group, first in order to understand the disease quickly, we drew anatomical axis of tibia and angles of ankle as same as the CO group (Figs. 1a and 1b). Second, the CT data were entered into E-3D V17.08 software (Huiqing, LTD., Nanjing, China) and was built into a 3D digital model, then we performed a simulated operation on the digital model (Fig. 1d). The simulated operation was usually divided into five steps: (1) We drew the axis and angles on the digital model to understand the disease further. (2) We did osteotomy, reduction and other special operative step on the digital model, at this point, we could repeatedly adjust the osteotomy, reduction and special operative step until the parameters became the most perfect (Fig. 1c). (3) We chose one to four suitable plates and appropriate screws from our virtual database of internally fixed models, after that, we installed the virtual internal fixation on the digital model. (4) We designed a PSI to help with the operation; usually we created an osteotomy guide plate to assist in osteotomy, and a temporary fixation guide plate to maintain the normal position of bone blocks.

(5) We checked repeatedly to see if the simulated operation was satisfactory, and did some actively modified until it became perfect. Third, after satisfactory simulated operation, all procedures, tools and internal fixation were recorded and prepared accordingly. In addition, we used nylon material to construct the PSI and life-sized 3D model, using the selective laser sintering (SLS) technique (Fig. 1e).

\section{Operation and rehabilitation}

In the CO group, as same as the conventional SMOT [1, 2, 7, 15-17], the medial point of osteotomy line was about $3 \mathrm{~cm}$ to $5 \mathrm{~cm}$ above the joint surface. According to the preoperative plan, we did osteotomy and other steps to perfect the ankle alignment and position assisted by multiple intraoperative fluoroscopies.

In the SO-PSI group, most steps were as same as the CO group, but there were still differences. (1) The position of the osteotomy line was determined, according to the osteotomy guide plate; then an osteotomy could be performed accurately without repeated confirmations and attempts (Fig. 1f). (2) Perfect axes and angles were obtained through the assistance of temporary fixation guide plate; when the plate was installed on bone blocks and then form a rigid whole reciprocally, the ankle alignment and position will be consistent with the preoperative plan (Figs. $1 \mathrm{~d}$ and $1 \mathrm{~g}$ ). (3) Suitable plates and appropriate screws were installed on skeleton according to the preoperative plan (Fig. 1d). The ankle alignment and position in the mortise were verified fluoroscopically before and after final fixation.

For both two groups, the patient wore a plaster slab until the stitch of wound was removed, then wore a below-knee protective plaster cast. Patients were encouraged to mobilize partially weight bearing for the first 3-6 weeks; after that, full weight-bearing without plaster cast began after the osteotomy site reached bony union radiographically.

\section{Measurement and follow-up}


To compare the difference between the two groups during the perioperative period, we carefully searched and collected: pre-op planning time, number of plate, op-special step, op-time, number of fluoroscopies, op-blood loss, reduction of albumin (ALB, the difference of albumin value between pre-op two days and post-op two days), total hospital stay, hospitalization expenses, complication rate and union time.

To evaluate the accuracy of preoperative planning and intraoperative application, preoperative planning and postoperative three to six months actual imaging data were compared. In CO group, we compared the TASs and TLSs; in SO-PSI group, we compared the TASs, TTAs, MAs, TLSs and tibial ankle center discrepancy (TACD, the distance between tibial ankle center of preoperative SO plan data and actual postoperative three to six months CT data) (Figs. $1 \mathrm{~h}$ and $1 \mathrm{j}$ ).

To compare the postoperative follow-up of the two groups, we carefully searched and collected the imaging data to compare the changes of the ankle angles and Takakura stage. Moreover, the American Orthopaedic Foot \& Ankle Society (AOFAS) ankle-hindfoot score [18], range of motion (ROM) of ankle [19] and visual analogue scale (VAS) [20]were used to evaluate the functional outcomes pre-and postoperation.

\section{Statistical analysis}

Independent Samples t-test was used to analyze the difference of the age, BMI, AOFAS score, VAS, ROM of ankle, follow-up time, pre-op planning time, op-time, number of fluoroscopies, op-blood loss, reduction of ALB, hospital stay, pre-op hospital stay, post-op hospital stay, hospitalization expenses and union time. Chi-squared test was used to analyze the difference of the gender, etiology, diabetes, smoker, Takakura stage, number of plate, special op-step and complication rate. Independent-samples t-test was used to analyze the changes of angles before and after operation. Linear correlation regression analysis was used to analyze the accuracy of pre-op plans in both two groups. Significance was defined as $p<0.05$. Statistical analysis was performed with SPSS 20.0 software (SPSS Inc., Chicago, IL, USA).

\section{Results}

\section{Perioperative outcomes}

The pre-op planning time of SO-PSI group $(289.1 \pm 44.4 \mathrm{~min})$ was significantly greater than the CO group $(31.5 \pm 9.1 \mathrm{~min})(P=0.000)$. The op-time of SO-PSI group $(101.8 \pm 16.0 \mathrm{~min})$ was significantly less than the $\mathrm{CO}$ group $(134.1 \pm 23.2 \mathrm{~min})(P=0.000)$. The number of fluoroscopies of SO-PSI group $(7.4 \pm 2.1)$ was significantly less than the $\mathrm{CO}$ group $(10.3 \pm 3.3)(P=0.008)$. The reduction of ALB of SO-PSI group $(1.3 \pm$ $0.3 \mathrm{~g} / \mathrm{L})$ was significantly less than the $\mathrm{CO}$ group $(2.7 \pm 1.1 \mathrm{~g} / \mathrm{L})(P=0.000)$. The pre-op hospital stay of SO-PSI group ( $5.5 \pm 1.4$ days) was significantly greater than the $C O$ group $(2.0 \pm 0.8$ days $)(P=0.000)$; but the post-op hospital stay of SO-PSI group ( $6.6 \pm 1.8$ days) was significantly less than the CO group (9.2 \pm 1.7 days) $(P=0.001)$. The hospitalization expenses of SO-PSI group $(11369.1 \pm 1700.3$ dollars $)$ was significantly greater than the $\mathrm{CO}$ group $(9888.2 \pm 1472.6$ dollars $)(P=0.028)$. The other parameters of two groups have no significant difference. (Table 2) 
Table 2

Perioperative outcomes.

\begin{tabular}{|clll|}
\hline & $\begin{array}{l}\text { CO group } \\
(\mathbf{n}=17)\end{array}$ & $\begin{array}{l}\text { SO-PSI group } \\
(\mathbf{n}=11)\end{array}$ & P values \\
\hline Mean of pre-op planning time (min) & $31.5 \pm 9.1$ & $289.1 \pm 44.4$ & 0.000 \\
\hline Number of plate (No. and \%) & & & \\
\hline One plate & $3(17.6 \%)$ & $1(9.1 \%)$ & 0.527 \\
\hline Two plates & $5(29.4 \%)$ & $3(27.3 \%)$ & 0.903 \\
\hline Three plates & $6(35.3 \%)$ & $3(27.3 \%)$ & 0.657 \\
\hline Four plates & $3(17.6 \%)$ & $4(36.4 \%)$ & 0.264 \\
\hline Special op-step (No. and \%) & & & 0.736 \\
\hline Calcaneal osteotomy & $13(76.5 \%)$ & $9(81.8 \%)$ & 0.903 \\
\hline Fore/mid-foot osteotomy & $5(29.4 \%)$ & $3(27.3 \%)$ & 0.823 \\
\hline Tibia lengthening & $2(11.8 \%)$ & $1(9.1 \%)$ & 0.747 \\
\hline Other & $1(5.9 \%)$ & $1(9.1 \%)$ & 0.000 \\
\hline Mean of op-time (min) & $134.1 \pm 23.2$ & $101.8 \pm 16.0$ & 0.008 \\
\hline Mean of number of fluoroscopies & $10.3 \pm 3.3$ & $7.4 \pm 2.1$ & 0.076 \\
\hline Mean of op-blood loss (ml) & $104.5 \pm 41.6$ & $139.2 \pm 54.6$ & 0.000 \\
\hline Mean of reduction of ALB (g/L) & $2.7 \pm 1.1$ & $1.3 \pm 0.3$ & 0.132 \\
\hline Mean of hospital stay (days) & $11.2 \pm 1.6$ & $12.2 \pm 1.5$ & 0.000 \\
\hline Mean of pre-op hospital stay (days) & $2.0 \pm 0.8$ & $5.5 \pm 1.4$ & $6.6 \pm 1.8$ \\
\hline Mean of post-op hospital stay (days) & $9.2 \pm 1.7$ & $11369.1 \pm 1700.3$ & \\
\hline Mean of hospitalization expenses (dollars) & $9888.2 \pm 1472.6$ & \\
\hline
\end{tabular}

Abbreviations: CO: Conventional operation group; SO-PSI: Simulated operation combines Patientspecific guide; Pre-op: preoperative; Op: operative; Reduction of ALB: The difference of albumin value between preoperative 2 days and postoperative 2 days; Post-op: postoperative.

\# $P$ values, $a=0.05$, (Pre-op planning time, op-time, number of fluoroscopies, op-blood loss, reduction of ALB, hospital stay, pre-op hospital stay, post-op hospital stay, hospitalization expenses and union time: independent-samples t-test; Number of plate, Special op-step and Complication rate: Chisquared test)

* Four patients of $\mathrm{CO}$ group and one patient of SO-PSI group occurred delayed wound healing after operation, and all of them have healed completely 3 to 5 weeks after operation. 


\begin{tabular}{|llll|}
\hline & $\begin{array}{l}\text { CO group } \\
(\mathbf{n}=17)\end{array}$ & $\begin{array}{l}\text { SO-PSI group } \\
(\mathbf{n}=11)\end{array}$ & P values \\
\hline Complication* rate (no. and \%) & $4(23.5 \%)$ & $1(9.1 \%)$ & 0.330 \\
\hline Mean of union time (weeks) & $14.1 \pm 1.2$ & $13.5 \pm 0.8$ & 0.201 \\
\hline $\begin{array}{l}\text { Abbreviations: CO: Conventional operation group; SO-PSI: Simulated operation combines Patient- } \\
\text { specific guide; Pre-op: preoperative; Op: operative; Reduction of ALB: The difference of albumin value } \\
\text { between preoperative 2 days and postoperative 2 days; Post-op: postoperative. }\end{array}$ & \\
\hline $\begin{array}{l}\text { \# P values, a = 0.05, (Pre-op planning time, op-time, number of fluoroscopies, op-blood loss, reduction } \\
\text { of ALB, hospital stay, pre-op hospital stay, post-op hospital stay, hospitalization expenses and union } \\
\text { time: independent-samples t-test; Number of plate, Special op-step and Complication rate: Chi- } \\
\text { squared test) }\end{array}$ & & \\
\hline $\begin{array}{l}\text { * Four patients of co group and one patient of SO-PSI group occurred delayed wound healing after } \\
\text { operation, and all of them have healed completely 3 to 5 weeks after operation. }\end{array}$ & \\
\hline
\end{tabular}

\section{Accuracy of preoperative planning and intraoperative application}

Through postoperative imaging examination, all cases can be found the ankle alignments and positions have been recovered to varying degrees (Fig. 2). In $\mathrm{CO}$ group, there were bad correlation between pre-op planning and post-op three to six months were seen for TAS $\left(R^{2}=0.100, P=0.271\right)$ and TLS $\left(R^{2}=0.111\right.$, $P=0.192$ ) (Figs. 3a and $3 b$ ). But in SO-PSI group, there were good correlation between pre-op planning and post-op three to six months were seen for TAS $\left(R^{2}=0.667, P=0.002\right)$, TTA $\left(R^{2}=0.438, P=0.027\right)$, MA $\left(R^{2}=0.709, P=0.001\right)$ and TLS $\left(R^{2}=0.925, P=0.000\right)$ (Figs. 3c-3f); Moreover, the mean TACD of SO-PSI group was $1.86 \pm 1.06 \mathrm{~mm}$ (range, 0.51 to $4.2 \mathrm{~mm}$ ) (Table 3 ). 
Table 3

Imaging parameters outcomes.

\begin{tabular}{|c|c|c|c|}
\hline & $\begin{array}{l}\text { CO group } \\
(n=17)\end{array}$ & $\begin{array}{l}\text { SO-PSI group } \\
(n=11)\end{array}$ & P values $\#$ \\
\hline $\begin{array}{l}\text { Mean of tibial ankle center discrepancy* } \\
\text { (TACD, mm) (post-op } 3 \text { to } 6 \text { months) }\end{array}$ & $\mathrm{N} / \mathrm{A}$ & $1.86 \pm 1.06$ & $\mathrm{~N} / \mathrm{A}$ \\
\hline \multicolumn{4}{|l|}{ Mean of TAS $\left(^{\circ}\right)$} \\
\hline Pre-op & $80.1 \pm 4.7$ & $74.4 \pm 10.5$ & 0.118 \\
\hline Post-op 1 year & $87.0 \pm 2.6$ & $89.5 \pm 1.8$ & 0.006 \\
\hline$P$ value (Paired-samples t-test) & 0.009 & 0.000 & \\
\hline \multicolumn{4}{|l|}{ Mean of TTA $\left(^{\circ}\right)$} \\
\hline Pre-op & $7.4 \pm 3.3$ & $6.6 \pm 2.7$ & 0.513 \\
\hline Post-op 1 year & $3.8 \pm 2.7$ & $1.4 \pm 1.5$ & 0.005 \\
\hline$P$ value (Paired-samples t-test) & 0.002 & 0.000 & \\
\hline \multicolumn{4}{|l|}{ Mean of MA $\left(^{\circ}\right)$} \\
\hline Pre-op & $75.3 \pm 3.5$ & $74.4 \pm 3.6$ & 0.488 \\
\hline Post-op 1 year & $84.3 \pm 2.4$ & $85.7 \pm 1.9$ & 0.097 \\
\hline$P$ value (Paired-samples t-test) & 0.000 & 0.000 & \\
\hline \multicolumn{4}{|l|}{ Mean of TLS $\left(^{\circ}\right)$} \\
\hline Pre-op & $73.8 \pm 3.3$ & $75.1 \pm 3.9$ & 0.371 \\
\hline Post-op 1 year & $78.8 \pm 2.9$ & $79.2 \pm 4.7$ & 0.816 \\
\hline$P$ value (Paired-samples t-test) & 0.000 & 0.000 & \\
\hline \multicolumn{4}{|c|}{$\begin{array}{l}\text { Abbreviations: CO: Conventional operation group; SO-PSI: Simulated operation combines Patient- } \\
\text { specific guide; TACD: Tibial ankle center discrepancy (the distance between tibial ankle center of } \\
\text { preoperative SO plan data and actual postoperative three to six months CT data); TAS: Tibial anterior } \\
\text { surface angle; TTA: Tibiotalar tilt angle; MA: Malleolar angle; TLS: Tibial lateral surface angle. }\end{array}$} \\
\hline \multicolumn{4}{|c|}{ \# $P$ values, $a=0.05$ (Independent-samples t-test) } \\
\hline
\end{tabular}

\section{Follow-up outcomes}

All patients were followed up, for radiologic evaluation at post-op one year, all of the included parameters of both two groups significantly improved. But the improvement of TAS and TTS of SO-PSI group were 
more obvious than that of $\mathrm{CO}$ group (TAS: $\mathrm{P}=0.006$, TTS: $\mathrm{P}=0.005$ ) (Table 3 ). The AOFAS score of SOPSI group and $\mathrm{CO}$ group increased from $51.7 \pm 10.9$ and $52.5 \pm 10.6$ before operation to $88.2 \pm 5.7$ and $83.1 \pm 4.5$ respectively at the final follow-up; The AOFAS score of SO-PSI group were better than that of CO group post-op six months $(P=0.014)$, one year $(P=0.048)$ and final follow-up $(P=0.024)$ (Fig. $4 a)$. The VAS of SO-PSI group and CO group decreased from $7.3 \pm 1.6$ and $7.7 \pm 1.4$ before operation to $3.7 \pm$ 0.7 and $3.1 \pm 0.9$ respectively at the final follow-up; The VAS of SO-PSI group was only better than that of $\mathrm{CO}$ group post-op six months $(\mathrm{P}=0.006)$ (Fig. $4 \mathrm{~b})$. The ROM of ankle of SO-PSI group and $\mathrm{CO}$ group increased from $31.8 \pm 8.4$ and $31.2 \pm 6.8$ before operation to $37.8 \pm 4.4$ and $35.9 \pm 5.5$ respectively at the final follow-up; The ROM of ankle between two groups has no significant difference at each time point (Fig. 4c). Takakura stage of both two groups improved after the operation, but the improvement of SO-PSI group seemed to be better than that in $\mathrm{CO}$ group. In $\mathrm{CO}$ group at the final follow-up, compared to preoperative four patients improved their Takakura stage by two levels $(23.5 \%)$, nine patients by one level (52.9\%), three patients had similar stage (17.6\%) and one patient became worse (5.9\%) (Fig. 5a). In SOPSI group at the final follow-up, compared to preoperative six patients improved their Takakura stage by two levels (54.5\%), four patients by one level (36.4\%) and one patient had similar stage (9.1\%) (Fig. 5b).

\section{Discussions}

This study aimed to determine whether there is a difference between CO and SO-PSI technology in terms of preoperative planning, intraoperative application and postoperative follow-up for SMOT. Referring to relevant studies and combining with own experience, it is strongly suggested that accurate preoperative analysis of condition and the ideal postoperative ankle angles, line of force and arthritis stage are very important for SMOT, which has also become the focus of current research $[1,2,6-10,15-17,21]$.

Analyzing results of the study, we found that SO-PSI technology have advantage over CO technology in preoperative planning, intraoperative application and postoperative follow-up. There should be four reasons: (1) Preoperative 3D analysis on computer can accurately understand original state of the disease, which is much more reliable than the conventional hand-painted two-dimensional analysis and simple observation of three-dimensional reconstruction of CT images; many studies have reached similar conclusions, and it is generally believed that preoperative 3D digital analysis will greatly deepen surgeons' cognition of the original state of the disease [22, 23]. (2) Still consistent with similar studies, the surgeon has performed repeated simulated operations on a digital model before surgery, they must to face difficulties and detail ready prepared ahead of time; at this time the feasibility and perfectness of the preoperative planning are preferable because it is well thought out [24]. (3) Compared with conventional technology, the use of PSI in operation can make the traditional osteotomy and alignment process faster and more accurate; relevant studies have shown that PSI can reduce the number of surgical procedures and fluoroscopies during the operation, and with the help of PSI, many procedures no longer require repeated attempts [11]. (4) Another possible reason is that our team's rich digital orthopedic operation experience [12] provides this new technology with good personnel, technical reserves and execution ability. 
However, there are also shortcomings of the SO-PSI technology: Firstly, the preoperative planning time of SO-PSI group was much longer than that of the $\mathrm{CO}$ group in the study, which is due to the addition of many preoperative steps, including copying the original CT data, 3D digital model reconstruction, simulated operation completion and PSI 3D printing. These steps are very time-consuming [25]. Secondly, the average hospitalization expenses of SO-PSI group were 1,400 dollars higher than that of the CO group in the study; the reason is that the designer labor fee and 3D printing production fee need to be increased, and it is not included in the medical insurance in our country. However, with the improvement of health care policies, software upgrades and rapid development of 3D printing technology [26, 27], it is gradually getting easier and faster for doctors to complete these steps, and the corresponding costs will gradually drop too.

Moreover, there are some limitations: First, designing the PSI is a complex procedure, usually the surgeon needs a technologist who is skilled at the software to assist to make the PSI. Second, it is necessary to research the survival rate of ankle joint in each group after surgery on the basis of long-term observation in the future. Third, this study is only a retrospective study; a prospective study should be conducted, even randomized controlled trial.

\section{Conclusions}

Our results indicate that SO-PSI technology can carry out the preoperative plan accurately and quickly in SMOT; In general, compared with the conventional technology, SO-PSI technology has advantages in preoperative planning, intraoperative application and postoperative curative effect.

\section{Abbreviations}

SO-PSI

simulated operation combines patient-specific guide

SMOT

supramalleolar osteotomy

CO

conventional operation

AOFAS

American Orthopaedic Foot \& Ankle Society

VAS

visual analogue scale

3D

three-dimensional

BMI

body mass index

TAS

tibial anterior surface angle 
TTA

tibiotalar tilt angle

MA

malleolar angle

TLS

tibial lateral surface angle

SLS

selective laser sintering

ALB

albumin

TACD

tibial ankle center discrepancy

ROM

range of motion

\section{Declarations}

\section{Acknowledgements}

This study was strongly supported by the Digital Research Institute of Orthopedics of Xiangya Hospital, the Ethics Committee of Xiangya Hospital, the registration system of foot and ankle surgery department, the medical record information system and the follow-up system of patients' service center of Xiangya Hospital.

\section{Availability of data and materials}

The datasets used and/or analyzed during the study are available from the corresponding author upon reasonable request.

\section{Authors' contributions}

Liu.H and Wang.CG contributed to the investigation, methodology, data curation, and preparation of the original draft. Xu.C and Li.YS contributed to the formal analysis, data curation, and preparation of the original draft. Xu.C and Wang.CG prepared the original draft. Zhong.D, Wang $L$ and Li.MQ did the validation of data. Liu.H and Wang.CG contributed to the design of the SO-PSI. Zhong.D and Liu.H contributed to the surgical guidance. Liu.H performed all the operations as the leader. Liu.H and Wang.CG edited and reviewed the manuscript. All authors read and approved the final manuscript.

\section{Conflicts of interest/Competing interests}

The authors declare that they have no conflict of interest.

Ethics approval and consent to participate 
This study has been approved by the ethics committee of our hospital (No.201906012). All procedures performed in studies were in accordance with the ethical standards of our institutional ethical committee. Informed consent was obtained from all individual participants included in the study.

\section{Consent for publication}

All patients involved had given informed consent

\section{References}

1. Kobayashi H, Kageyama Y, Shido Y. Treatment of Varus Ankle Osteoarthritis and Instability With a Novel Mortise-Plasty Osteotomy Procedure. J Foot Ankle Surg. 2016;55:60-7.

2. Nüesch C, Huber C, Paul J, Henninger HB, Pagenstert G, Valderrabano V, et al. Mid- to Long-term Clinical Outcome and Gait Biomechanics After Realignment Surgery in Asymmetric Ankle Osteoarthritis. Foot Ankle Int. 2015;36:908-18.

3. Zhao H, Liang X, Li Y, Yu G, Niu W, Zhang Y. The role of fibular for supramalleolar osteotomy in treatment of varus ankle arthritis: a biomechanical and clinical study. J Orthop Surg Res. 2016;11:127.

4. Qu W, Xin D, Dong S, Li W, Zheng Y. Supramalleolar osteotomy combined with lateral ligament reconstruction and talofibular immobilization for varus ankle osteoarthritis with excessive talar tilt angle. J Orthop Surg Res. 2019;14:402.

5. Takakura Y, Tanaka Y, Kumai T, Tamai S. Low tibial osteotomy for osteoarthritis of the ankle. Results of a new operation in 18 patients. J Bone Joint Surg Br. 1995;77:50-4.

6. Haraguchi N, Ota K, Tsunoda N, Seike K, Kanetake Y, Tsutaya A. Weight-bearing-line analysis in supramalleolar osteotomy for varus-type osteoarthritis of the ankle. J Bone Joint Surg Am. 2015;97:333-9.

7. Lee WC, Moon JS, Lee K, Byun WJ, Lee SH. Indications for supramalleolar osteotomy in patients with ankle osteoarthritis and varus deformity. J Bone Joint Surg Am. 2011;93:1243-8.

8. Ahn TK, Yi Y, Cho JH, Lee WC. A cohort study of patients undergoing distal tibial osteotomy without fibular osteotomy for medial ankle arthritis with mortise widening. J Bone Joint Surg Am. 2015;97:381-8.

9. Hongmou Z, Xiaojun L, Yi L, Hongliang L, Junhu W, Cheng L. Supramalleolar Osteotomy With or Without Fibular Osteotomy for Varus Ankle Arthritis. Foot Ankle Int. 2016;37:1001-7.

10. Pagenstert G, Knupp M, Valderrabano V, Hintermann B. Realignment surgery for valgus ankle osteoarthritis. Oper Orthop Traumatol. 2009;21:77-87.

11. Jud L, Vlachopoulos L, Beeler S, Tondelli T, Fürnstahl P, Fucentese SF. Accuracy of three dimensionalplanned patient-specific instrumentation in femoral and tibial rotational osteotomy for patellofemoral instability. Int Orthop. 2020; 
12. Wang C, Xiao H, Yang W, Wang L, Hu Y, Liu H, et al. Accuracy and practicability of a patient-specific guide using acetabular superolateral rim during THA in Crowe II/III DDH patients: a retrospective study. J Orthop Surg Res. 2019;14:19.

13. Xing QQ, Zhong D, Pan YX, An SB, Wang CG, Su SL, et al. A Comparative Study of Patients' Subjective Feelings Toward Total Hip Arthroplasty with Patient-Specific Instruments and Traditional Total Hip Arthroplasty. Orthop Surg. 2020;12:269-76.

14. Siddiqui NA, Lamm BM. Digital planning for foot and ankle deformity correction: Evans osteotomy. J Foot Ankle Surg. 2014;53:700-5.

15. Kim YS, Park EH, Koh YG, Lee JW. Supramalleolar Osteotomy With Bone Marrow Stimulation for Varus Ankle Osteoarthritis: Clinical Results and Second-Look Arthroscopic Evaluation. Am J Sports Med. 2014;42:1558-66.

16. Tanaka Y. The concept of ankle joint preserving surgery: why does supramalleolar osteotomy work and how to decide when to do an osteotomy or joint replacement. Foot Ankle Clin. 2012;17:545-53.

17. Knupp M, Stufkens SA, Bolliger L, Barg A, Hintermann B. Classification and treatment of supramalleolar deformities. Foot Ankle Int. 2011;32:1023-31.

18. Schneider W, Jurenitsch S. Normative data for the American Orthopedic Foot and Ankle Society ankle-hindfoot, midfoot, hallux and lesser toes clinical rating system. Int Orthop. 2016;40:301-6.

19. Rabin A, Portnoy S, Kozol Z. The Association of Ankle Dorsiflexion Range of Motion With Hip and Knee Kinematics During the Lateral Step-down Test. J Orthop Sports Phys Ther. 2016;46:1002-9.

20. Naunheim MR, Dai JB, Rubinstein BJ, Goldberg L, Weinberg A, Courey MS. A visual analog scale for patient-reported voice outcomes: The VAS voice. Laryngoscope Investig Otolaryngol. 2020;5:90-5.

21. Zhao HM, Wen XD, Zhang Y, Liang JQ, Liu PL, Li Y, et al. Supramalleolar osteotomy with medial distraction arthroplasty for ankle osteoarthritis with talar tilt. J Orthop Surg Res. 2019;14:120.

22. Qiang $M$, Zhang K, Chen $Y$, Jia X, Wang $X$, Chen S, et al. Computer-assisted virtual surgical technology in pre-operative design for the reconstruction of calcaneal fracture malunion. Int Orthop. 2019;43:1669-77.

23. Lei PF, Su SL, Kong LY, Wang CG, Zhong D, Hu YH. Mixed Reality Combined with Three-Dimensional Printing Technology in Total Hip Arthroplasty: An Updated Review with a Preliminary Case Presentation. Orthop Surg. 2019;11:914-20.

24. Chen Y, Jia X, Qiang M, Zhang K, Chen S. Computer-Assisted Virtual Surgical Technology Versus Three-Dimensional Printing Technology in Preoperative Planning for Displaced Three and Four-Part Fractures of the Proximal End of the Humerus. J Bone Joint Surg Am. 2018;100:1960-8.

25. Mitsouras D, Liacouras P, Imanzadeh A, Giannopoulos AA, Cai T, Kumamaru KK, et al. Medical 3D Printing for the Radiologist. Radiographics. 2015;35:1965-88.

26. Zastrow M. 3D printing gets bigger, faster and stronger. Nature. 2020;578:20-3.

27. Wang J, Cai L, Xie L, Chen H, Guo X, Yu K. 3D printing-based Ganz approach for treatment of femoral head fractures: a prospective analysis. J Orthop Surg Res. 2019;14:338. 

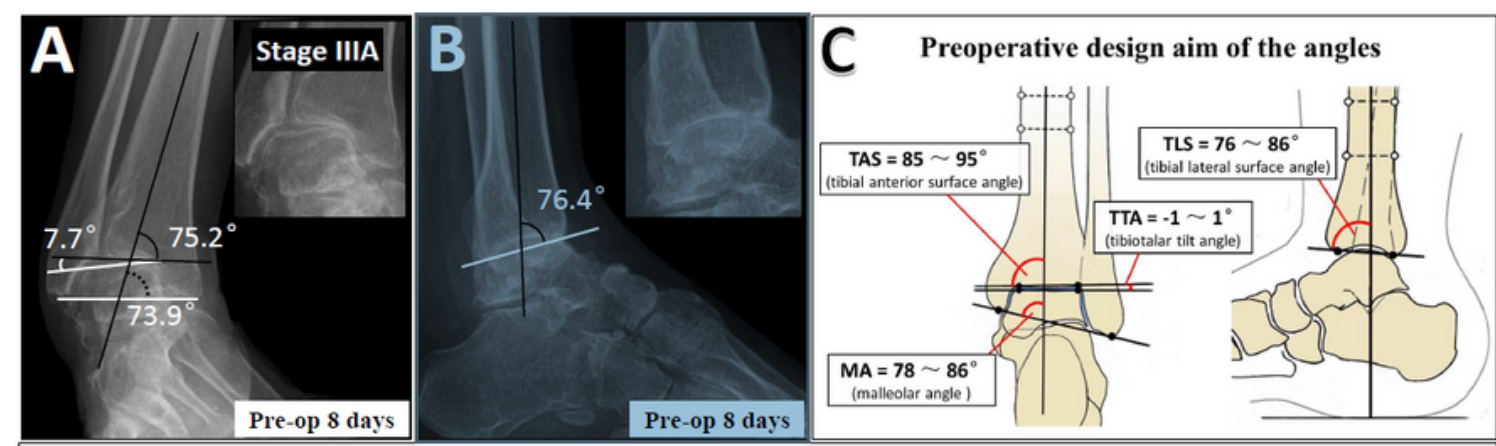

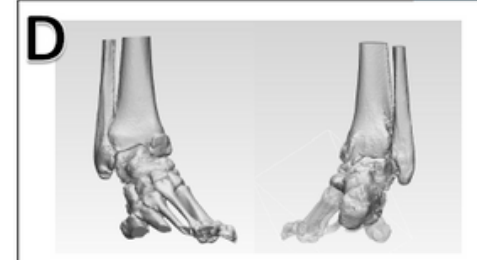

Pre-op CT

3D-reconstruction image

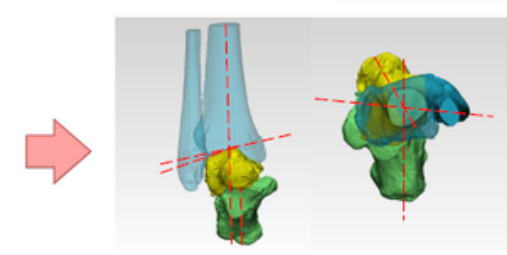

Analysis condition (pre-op simulated operation)

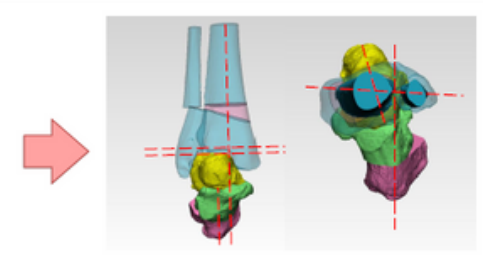

Osteotomy and reduction (pre-op simulated operation)

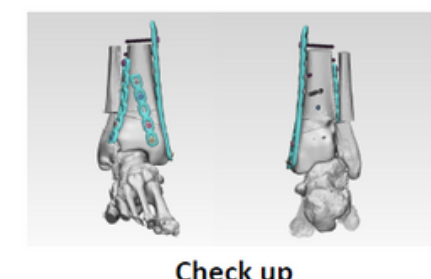

(Identify the ideal surgical procedure)

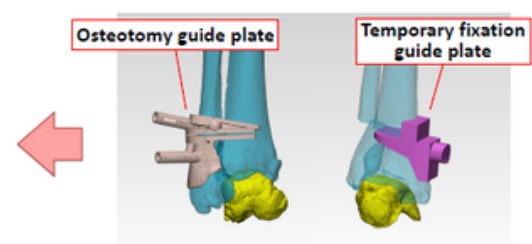

Design of PSI (usually consists of two parts)

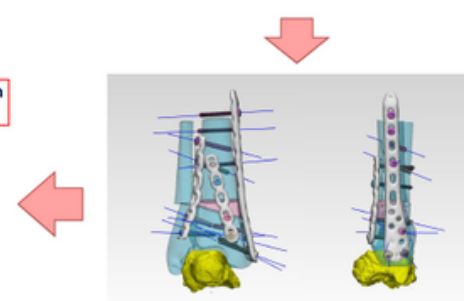

Internal fixation implantation (pre-op simulated operation)
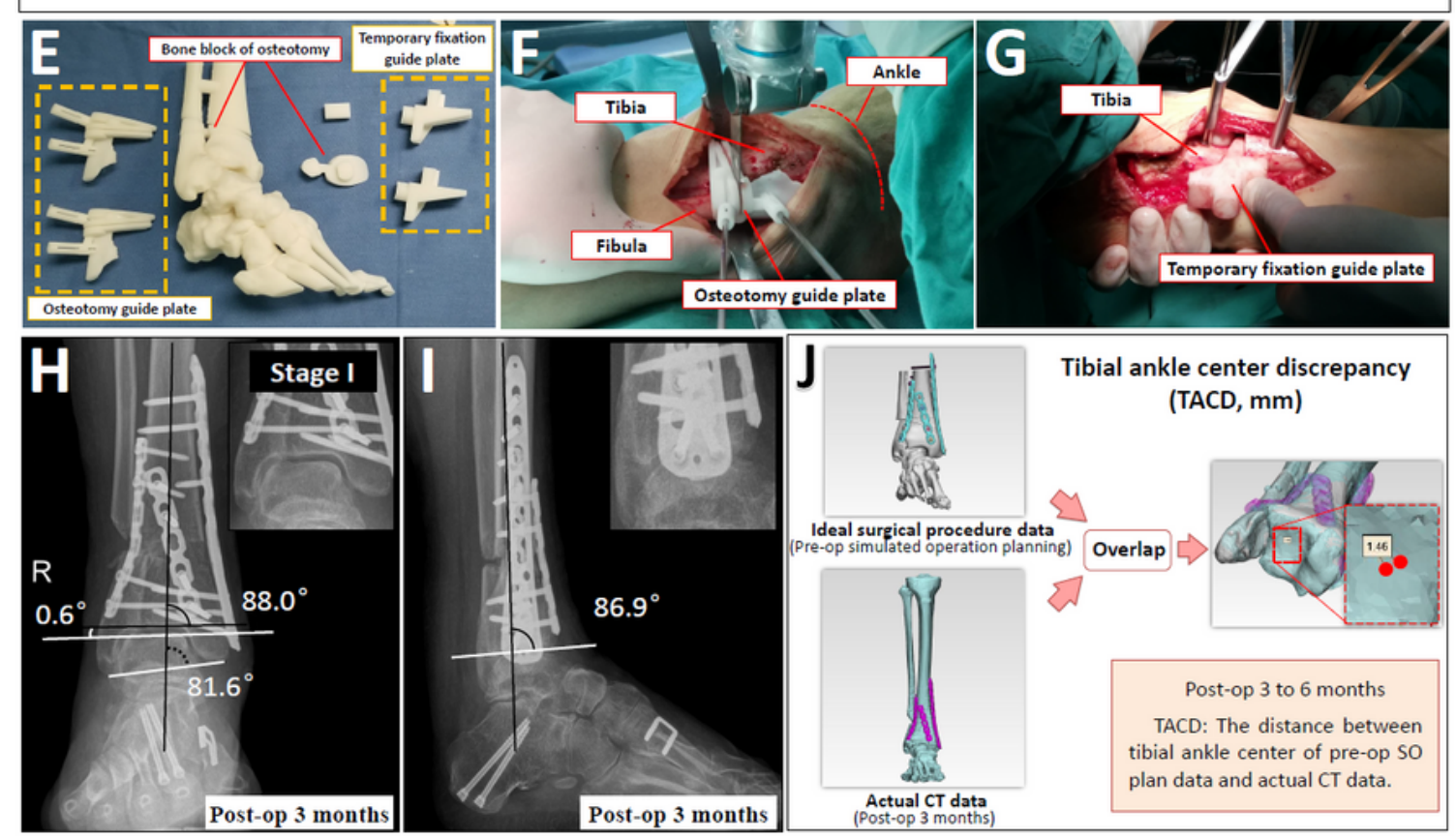

Figure 1

Application of SO-PSI technology for SMOT, but Fig. 1A-1C and Fig. $1 \mathrm{H}-1 \mathrm{I}$ are also suitable for conventional technology. (A, B) Measurement of relative Takakura stage, ankle angles and axes of (A) preoperative anteroposterior radiograph and (B) preoperative lateral radiograph. (C) Preoperative design 
aim of the angles. (D) Flow Chart of simulated operation. (E) Model and patient-specific guide made by 3D printing. (F) Application of osteotomy guide plate in operation. (G) Application of temporary fixation guide plate in operation. $(\mathrm{H}, \mathrm{I})$ Measurement of relative Takakura stage, ankle angles and axes of $(\mathrm{H})$ postoperative anteroposterior radiograph and (I) postoperative lateral radiograph. (J) TACD was measured after coincident preoperative planning and postoperative CT data.

\section{Typical case of $\mathrm{CO}$ group}

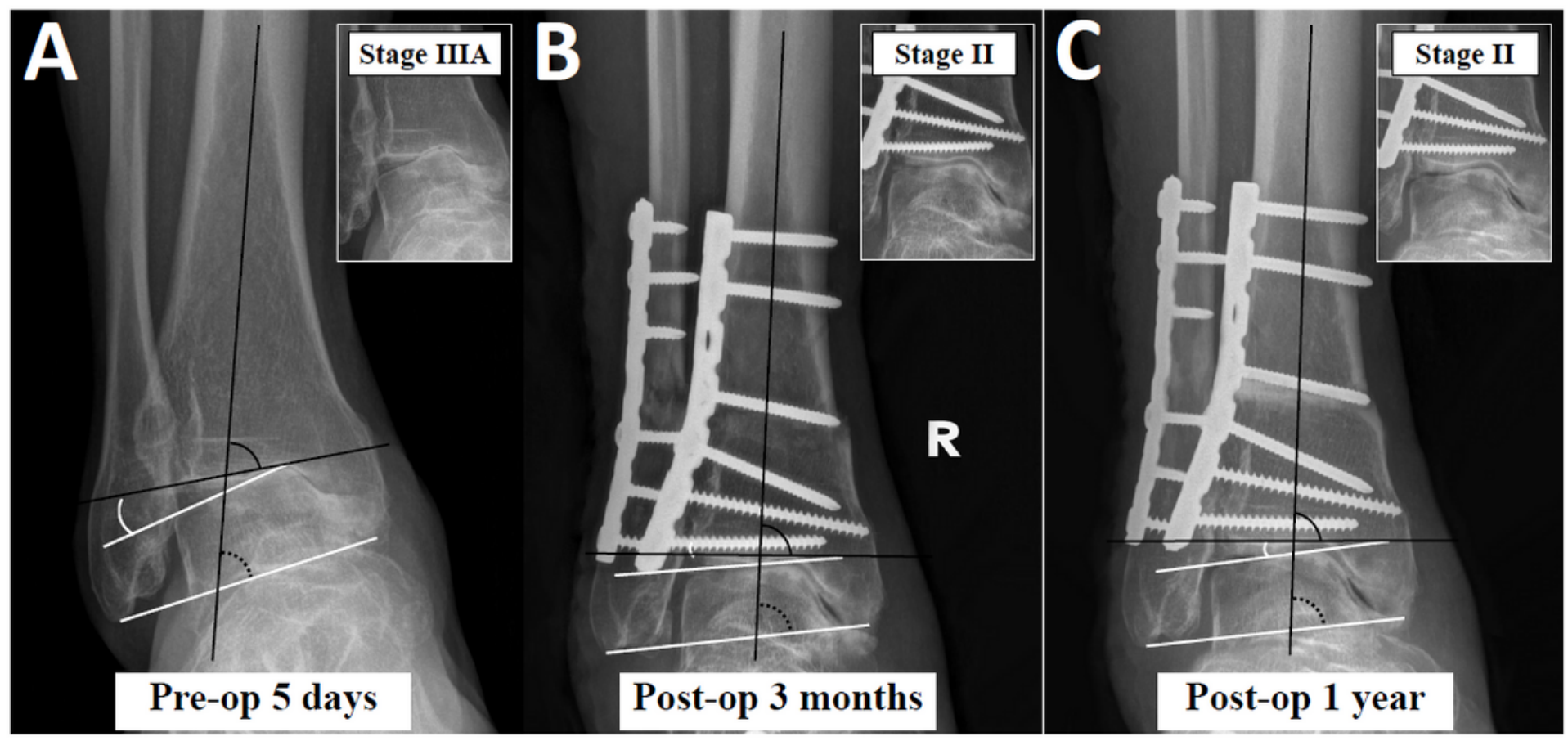

\section{Typical case of SO-PSI group}

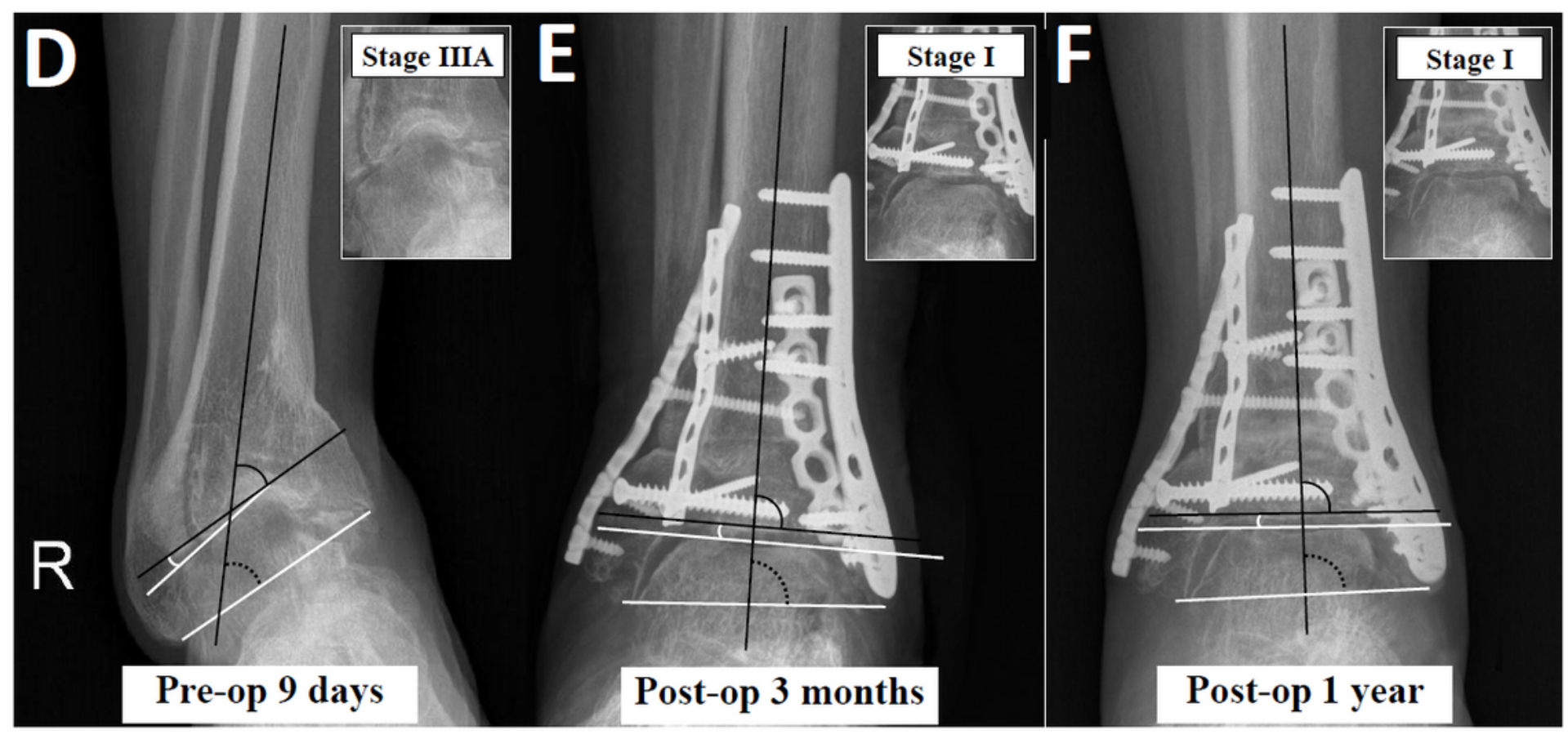

Figure 2 
X-ray of typical cases of two groups. (A-C) A Typical case of $\mathrm{CO}$ group: Anteroposterior radiograph of (A) preoperative five days, $(B)$ postoperative three months and (C) postoperative one year, and measurement of relative Takakura stage, ankle angles and axes. (D-F) A Typical case of SO-PSI group: Anteroposterior radiograph of $(D)$ preoperative nine days, $(E)$ postoperative three months and $(F)$ postoperative one year, and measurement of relative Takakura stage, ankle angles and axes.
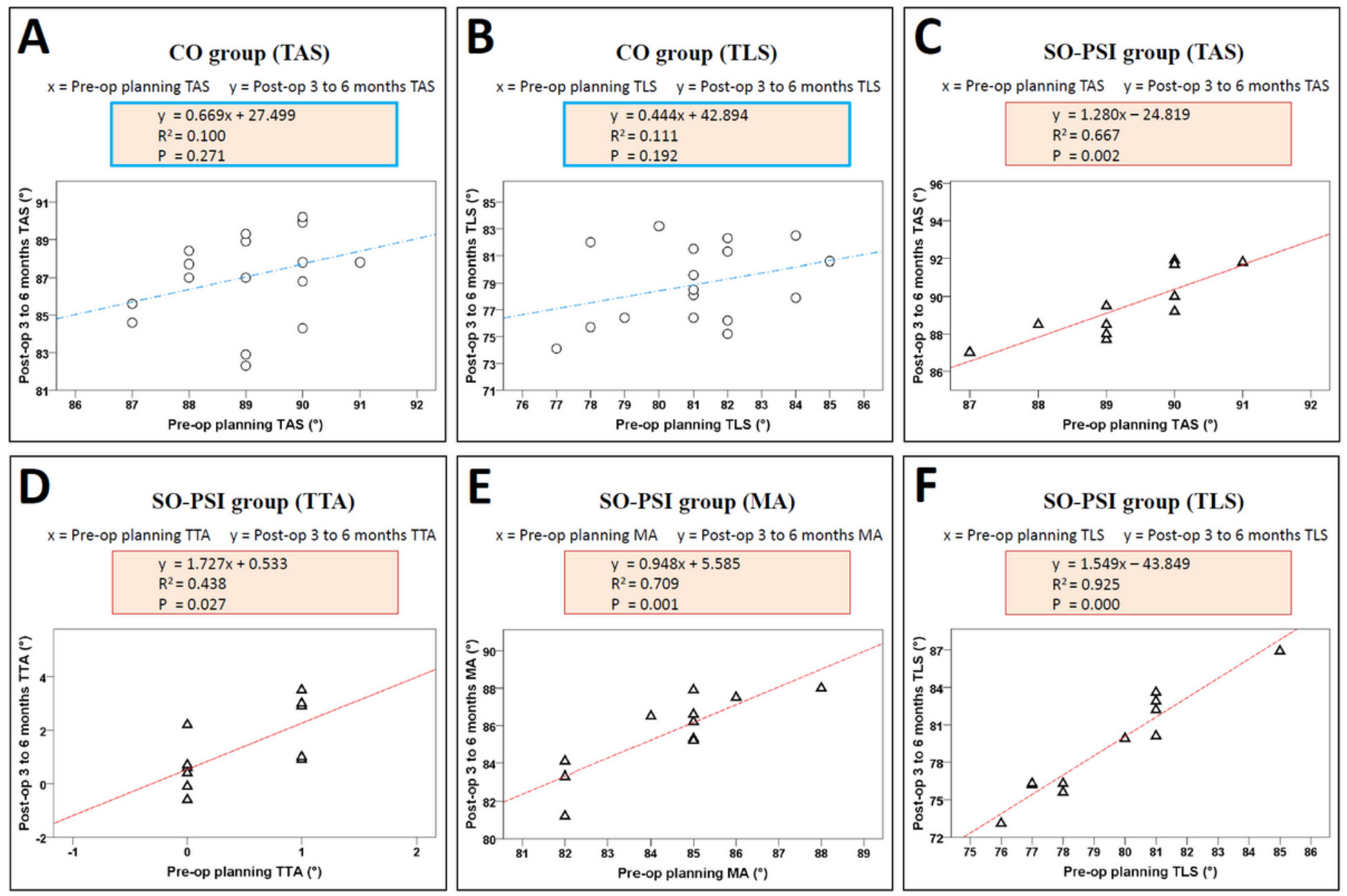

\section{Figure 3}

Preoperative planning and postoperative actual data linear regression analysis of two groups. $(A, B)$ Under this condition for $\mathrm{CO}$ group, the correlation of $(A)$ TAS $(R 2=0.100, P=0.271)$ and (B) TLS (R2=0.111, $P=0.192)$ were bad. (C-F) Under this condition for SO-PSI group, the correlation of (C) TAS (R2 $=0.667$, $P=0.002),(D)$ TTA (R2=0.438, $P=0.027),(E) M A(R 2=0.709, P=0.001)$ and $(F)$ TLS $(R 2=0.925, P=0.000)$ were all good. 

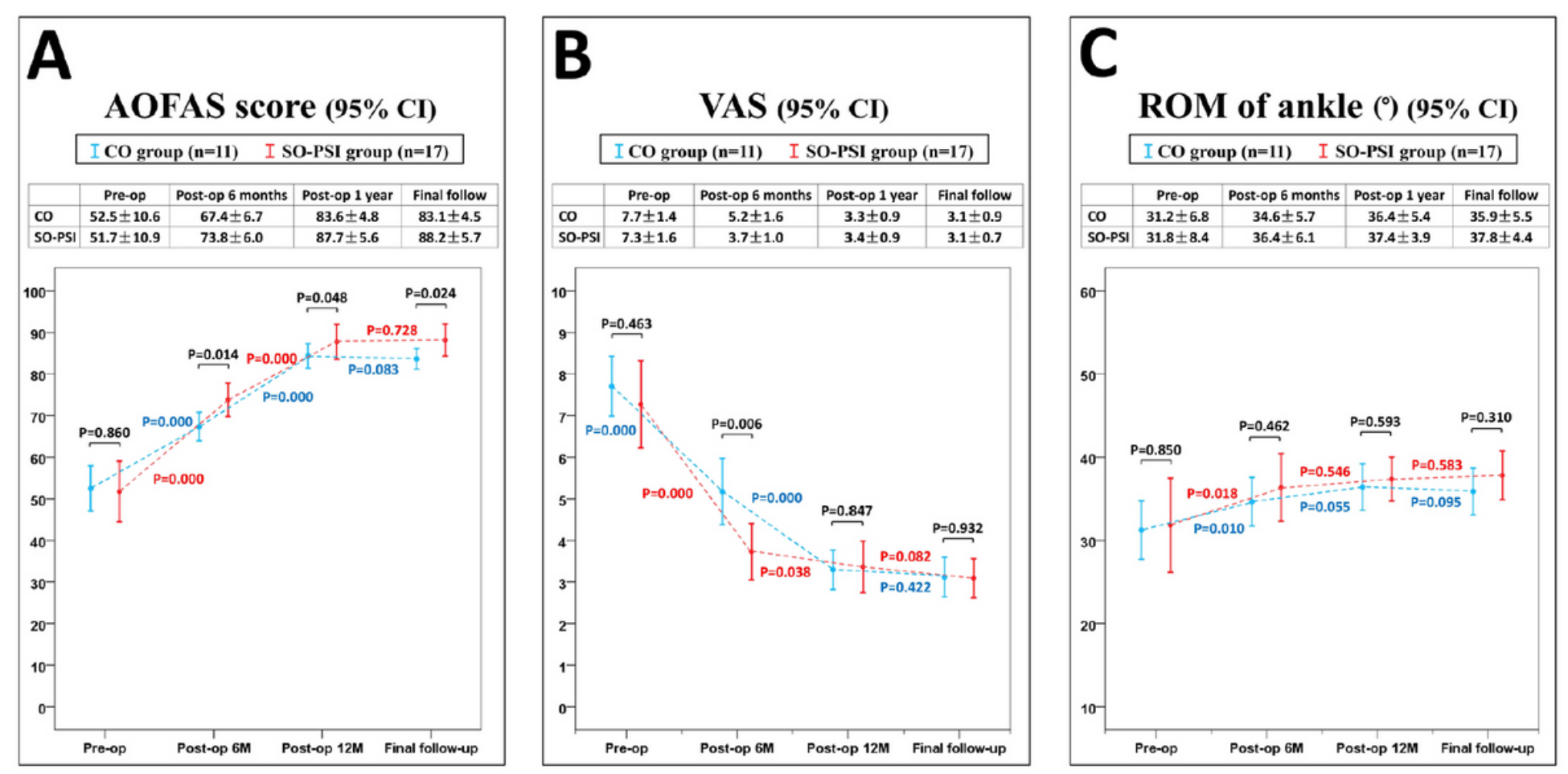

\section{Figure 4}

We can see from error bar charts that compared with CO group, AOFAS score, VAS and ROM of ankle of SO-PSI group gradually improved after operation. But specifically: (A) AOFAS score of SO-PSI group was better than that in CO group at each time point; (B) VAS of SO-PSI group was better than that in CO group postoperative six months; $(C)$ there was no significant difference in ROM of ankle between two groups at each time point. 


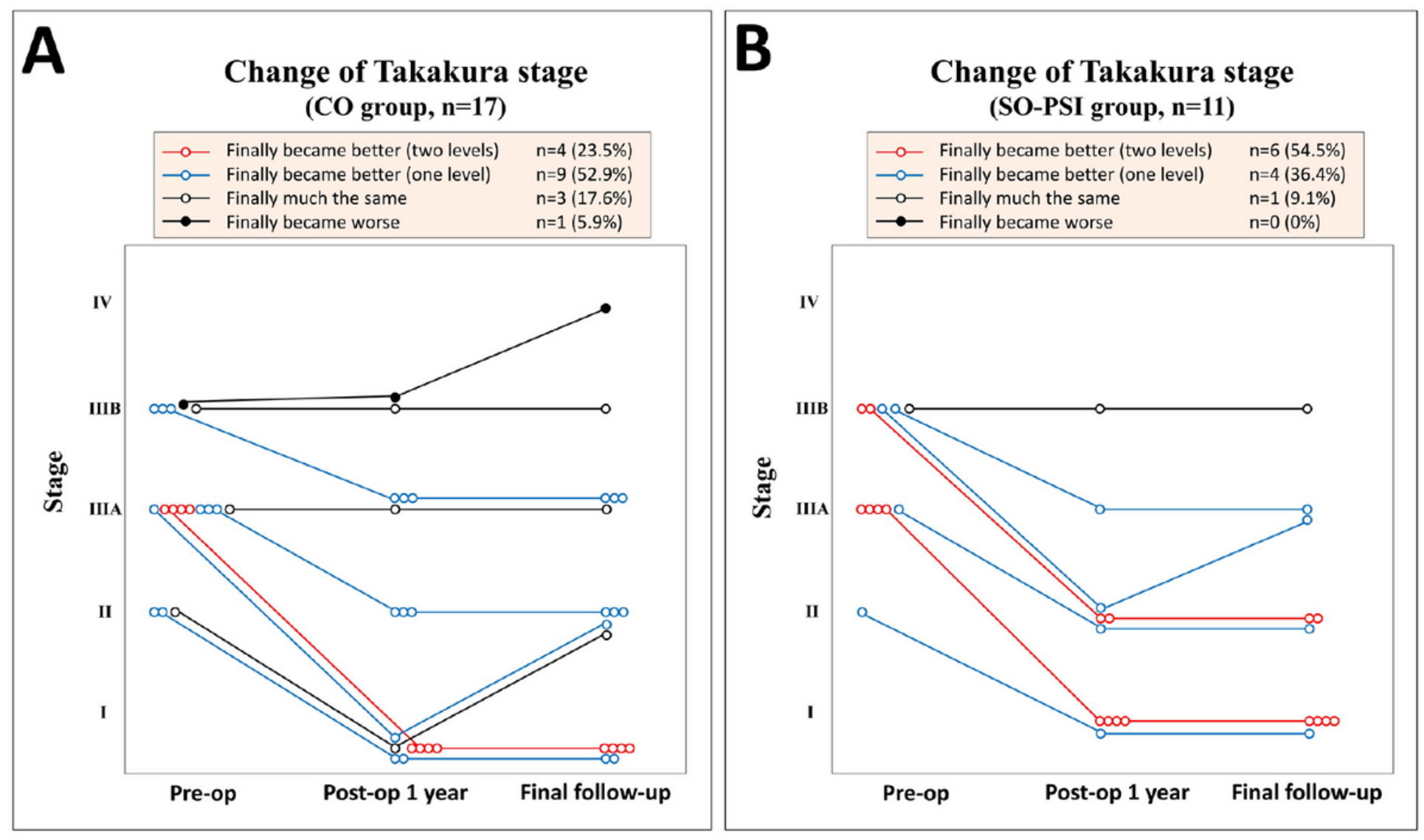

\section{Figure 5}

A graph plots the individual changes of Takakura stage of (A) CO group and (B) SO-PSI group with time respectively, each circle indicates one ankle (patient); Takakura stage of both two groups improved after the operation, but the improvement of SO-PSI group seemed to be better than that in CO group. 